\title{
Phosphorylation-dependent interaction between tumor suppressors Dlg and Lgl
}

\author{
Jinwei Zhu ${ }^{1, *}$, Yuan Shang ${ }^{1, *}$, Qingwen Wan ${ }^{3}$, Yitian Xia ${ }^{1}$, Jia Chen ${ }^{1,4}$, Quansheng Du ${ }^{3}$, Mingjie Zhang ${ }^{1,2}$ \\ ${ }^{I}$ Division of Life Science, State Key Laboratory of Molecular Neuroscience, Hong Kong University of Science and Technology, \\ Clear Water Bay, Kowloon, Hong Kong, China, ${ }^{2}$ Center of Systems Biology and Human Health, School of Science and Institute for \\ Advanced Study, Hong Kong University of Science and Technology, Clear Water Bay, Kowloon, Hong Kong, China, ${ }^{3}$ Department \\ of Neurology, Institute of Molecular Medicine and Genetics, Medical College of Georgia, Georgia Regents University, Augusta, \\ GA 30912, USA
}

The tumor suppressors Discs Large (Dlg), Lethal giant larvae (Lgl) and Scribble are essential for the establishment and maintenance of epithelial cell polarity in metazoan. Dlg, Lgl and Scribble are known to interact strongly with each other genetically and form the evolutionarily conserved Scribble complex. Despite more than a decade of extensive research, it has not been demonstrated whether Dlg, Lgl and Scribble physically interact with each other. Here, we show that Dlg directly interacts with Lgl in a phosphorylation-dependent manner. Phosphorylation of any one of the three conserved Ser residues situated in the central linker region of $\mathrm{Lgl}$ is sufficient for its binding to the Dlg guanylate kinase (GK) domain. The crystal structures of the Dlg4 GK domain in complex with two phosphorLgl2 peptides reveal the molecular mechanism underlying the specific and phosphorylation-dependent Dlg/Lgl complex formation. In addition to providing a mechanistic basis underlying the regulated formation of the Scribble complex, the structure of the $\mathrm{Dlg} / \mathrm{Lgl}$ complex may also serve as a starting point for designing specific Dlg inhibitors for targeting the Scribble complex formation.

Keywords: Dlg; Lgl; cell polarity; tumor suppressor; phosphorylation-dependent interaction; crystal structure Cell Research (2014) 24:451-463. doi:10.1038/cr.2014.16; published online 11 February 2014

\section{Introduction}

Cell polarity, a fundamental property of most animal cells, is essential for diverse cellular processes including cell proliferation, differentiation, asymmetric cell division, cell migration, tissue morphogenesis and tumor formation [1-5]. Loss of cell polarity is a hallmark of malignant tumors derived from epithelial tissues [6]. In the past several decades, elegant genetic and biochemical studies have identified three evolutionarily conserved complexes involved in the establishment and maintenance of the apical-basal cell polarity in the epithelial

*These two authors contributed equally to this work. Correspondence: Mingjie Zhang

E-mail: mzhang@ust.hk

${ }^{4}$ Current address: The Gurdon Institute, University of Cambridge, Tennis Court Road, Cambridge CB2 1QN, UK

Received 2 August 2013; revised 27 November 2013; accepted 13 January 2014; published online 11 February 2014 cells. These complexes are the Crumbs complex, the Par complex and the Scribble complex [7]. The Scribble complex proteins, composed of Discs Large (Dlg), Lethal giant larvae $(\mathrm{Lgl})$ and Scribble, were originally discovered as tumor suppressors in unbiased genetic screens in Drosophila [8-10]. Loss-of-function mutations in the three genes showed nearly identical phenotypes such as cell polarity defects, uncontrolled cell proliferation and loss of the proper cell architecture, which shares very similar characteristics in many epithelial-derived tumors in human $[3,6]$. Due to their strong genetic interactions, these three tumor suppressors are believed to function together in a common pathway to regulate cell polarity and to control tissue growth in epithelia [6].

However, despite the fact that the strong genetic interactions among Dlg, Lgl and Scribble have been discovered for more than a decade, the underlying molecular basis governing how the three proteins form a functional complex remains unclear and controversial. No conclusive evidence is available to show that $\mathrm{Dlg}$, 
$\mathrm{Lgl}$ and Scribble interact with each other directly to form a ternary complex or binary complexes. A protein called GUK-holder was reported to act as a linker between Dlg and Scribble in Drosophila [11]. The Nance-Horan syndrome (NHS) family-related protein Nhsl1b, a mammalian homolog of GUK-holder, was reported to coimmunoprecipitate with Dlg4 and Scribble in HEK293T cells $[12,13]$; however, the role of Nhsllb in apical-basal cell polarity is unknown. Although Lgl was shown to co-immunoprecipitate with Scribble when both proteins were overexpressed in HEK293T or MDCK cells [14], one cannot rule out the possibility that Lgl only associates with Scribble indirectly. In vivo imaging data have demonstrated that Dlg, Lgl and Scribble interdependently colocalize to the lateral membranes of epithelial cells and thereby specify the lateral membrane domain by excluding apical components from this region $[2,6]$. However, it is still not clear how $\mathrm{Lgl}$ is brought to the lateral membrane, although it is well established that apical atypical protein kinase $\mathrm{C}$ (aPKC) activity is required for excluding $\mathrm{Lgl}$ from apical membranes [15]. In dlg mutant embryos, Lgl basal membrane association is lost, indicating that Dlg may play a vital role in recruiting Lgl to the basal membranes [6]. All these observations suggest that the interactions between Dlg, Lgl and Scribble may be transient and subject to certain form(s) of regulation.

Here, we show that Lgl2 (a mammalian homolog of Drosophila $\mathrm{Lgl}$ ) directly interacts with $\mathrm{Dlg} 4$ (a mammalian homolog of Drosophila Dlg, also known as PSD-95) in vitro. The interaction between $\mathrm{Lgl} 2$ and $\mathrm{Dlg} 4$ strictly depends on the phosphorylation of at least one of the three phosphorylation sites situated in the central linker region of $\operatorname{Lg} 12$ by aPKC. The crystal structures of the Dlg4 guanylate kinase (GK) domain in complex with two phosphor-peptides comprising the GK-binding region of Lg12 reveal the general molecular mechanism underlying the $\mathrm{Dlg} / \mathrm{Lgl}$ complex formation. Our biochemical and structural results indicate that the formation of the Scribble complex is a regulated event that is tightly coupled to the establishment of cell polarity.

\section{Results}

The phosphorylation-dependent interaction between Lgl2 and Dlg4 GK domain

Earlier studies have demonstrated that the several closely spaced, highly conserved phosphorylation sites in the central linker region of $\mathrm{Lgl} 2$ can be phosphorylated by aPKC both in vivo and in vitro [16-19] (Figure 1A). Although phosphorylation is believed to be important for the proper function of Lg12, how the phosphorylation of $\mathrm{Lgl} 2$ affects its function is still unclear. We and others have demonstrated that the Dlg GK domain functions as a phosphor-peptide-binding module [20-22]. We therefore hypothesized that Dlg GK might also physically interact with $\mathrm{Lg} 12$ in a phosphorylation-dependent manner. We performed a GST pull-down assay to test whether aPKC-mediated phosphorylation of Lgl2 might regulate its binding to Dlg GK. As expected and consistent with failed attempts of observing direct interaction between Dlg and Lgl in the literature, GST-Dlg4 GK did not show detectable binding to HA-tagged Lg12 expressed in HEK293T cells (Figure 1B). Unexpectedly, GST-Dlg4 GK was found to bind robustly to HA-Lg12 when co-expressed with aPKC (Figure 1C). The Dlg4 GK-bound Lgl2 was immunoreactive to the anti-phosphor-Lgl antibody ( $\mathrm{p}-\mathrm{Lgl}$ ) that specifically recognizes the aPKC phosphorylation sites of Lgl2 (Figure 1A and 1C). As expected, due to the high amino-acid sequence identity between various isoforms of Dlgs, Dlg1 SH3GK also bound to Lg12 when aPKC was co-expressed in HEK293T cells (Supplementary information, Figure S1). Only background level of HA-Lg12 was pulled down by Dlg4 GK when a kinase-dead mutant of aPKC, in which the catalytic Lys 273 was replaced by Arg (K273R-aPKC), was co-expressed (Figure 1D). Additionally, aPKC could not be detected in the complex of Dlg4 GK and Lgl2 (Figure 1E). These data indicate that the interaction between Dlg4 GK and Lg12 is not mediated by aPKC. Taken together, the biochemical data shown in Figure 1 reveal that Lg12 can indeed be phosphorylated by aPKC and the phosphorylated Lg12 can specifically bind to Dlg4 GK directly.

As there are three evolutionarily conserved Ser residues (S645, S649 and S653) in Lg12 (Figure 1A), we next dissected which serine is involved in the binding to Dlg4 GK. We mutated each Ser individually to Ala and tested the binding of each Lg12 mutant to Dlg4 GK. Somewhat surprisingly, each single Lgl2 mutant (Lg12S645A, Lg12-S649A and Lg12-S653A) was found to bind Dlg4 GK as effectively as the wild-type Lgl2 (Figure 2A). As expected, the interaction between Lgl2 and Dlg4 GK was totally disrupted when all three Ser residues were substituted with Ala (Lg12-S645, 649, 653A, hereafter referred as to Lgl2 3A) (Figure 2B). Interestingly, a double mutant of Lg12 (Lg12-S645, 653A) displayed comparable binding to Dlg4 GK with the wild-type Lgl2 (Figure 2B). Two additional double mutants of $\mathrm{Lgl} 2$ (Lg12-S645, 649A and Lg12-S649, 653A) also showed similar binding to Dlg4 GK compared to the wild-type Lg12 (data not shown). A phosphomimetic mutant of Lg12 with the three Ser residues simultaneously substituted with Glu (Lg12-S645, 649, 653E) showed a detectable but much weaker binding to Dlg4 GK (Figure 2B), 
A

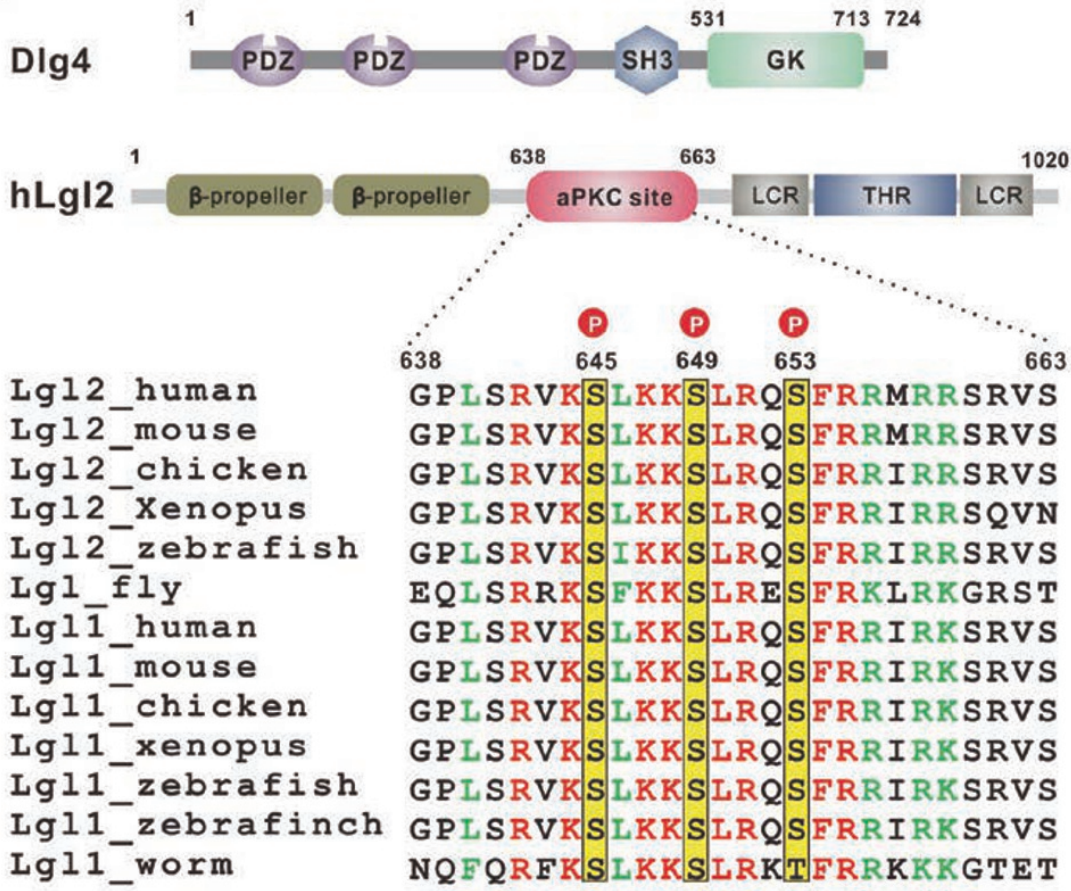

B

D

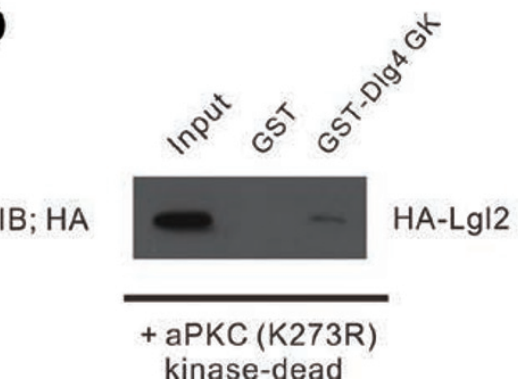

C

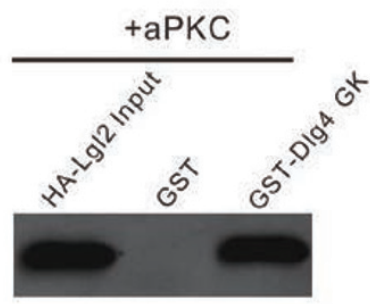

IB; $p-L g \mid$

E

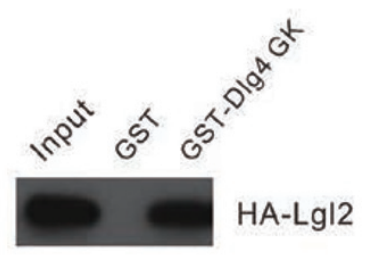

IB; Flag

Flag-aPKC

Figure 1 Phosphorylation-dependent binding of Lgl2 to Dlg4. (A) Schematic diagrams of the domain organizations of Dlg4 and Lgl2. The figure also illustrates the detailed boundaries of the GK domain (aa 531-713) used in this study as well as the aPKC phosphorylation sites (aPKC site, aa 638-663) on Lgl2. The amino-acid sequence alignment of the aPKC sites of Lgl from different species is also included. In this alignment, the absolutely conserved residues are colored in red, and the highly conserved residues are colored in green. (B, C) aPKC promotes the binding of Lgl2 to Dlg4 GK in the GST pull-down assays. Lgl2 binds to Dlg4 GK only in the presence of aPKC. The GK-bound Lgl2 proteins were also detected by the phosphor-Lgl antibody. (D, E) Kinase activity of aPKC is required for binding of Lgl2 to Dlg4. HA-Lgl2 cannot form complex with Dlg4 GK when a kinase-dead mutant of aPKC, K273R-aPKC, was co-expressed in HEK293T cells (D). Flag-aPKC cannot be detected in the complex of Dlg4 GK and HA-Lgl2 (E). 
A

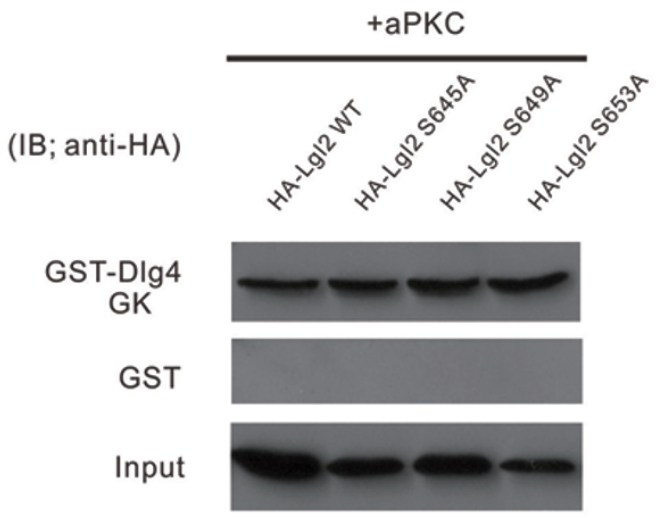

B
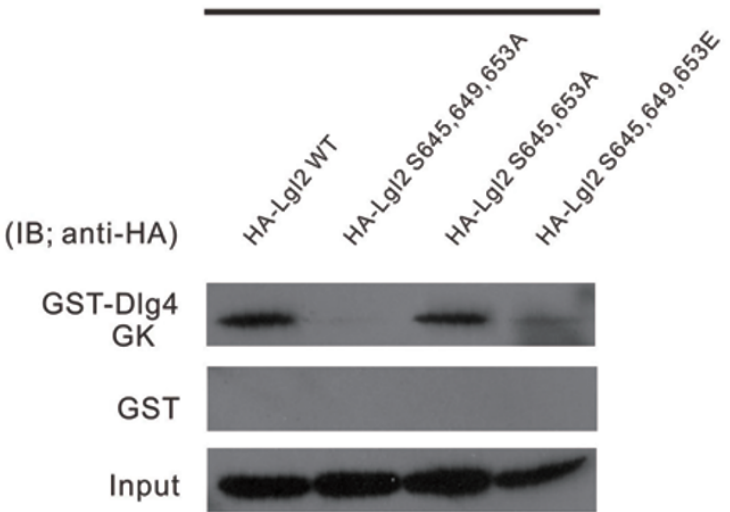

C

\begin{tabular}{|c|c|c|}
\hline Lgl peptide & Sequence & $\mathrm{Kd} / \mu \mathrm{M}$ \\
\hline & LS & $10.2 \pm 1.3$ \\
\hline$-\operatorname{Lg} 12 b:$ & ${ }^{643} \mathrm{VKSLKK}(\mathrm{PS}) \mathrm{L}$ & $12.2 \pm 1.3$ \\
\hline$p-\operatorname{Lg} 12 c:{ }^{64}$ & ${ }^{6} \mathrm{LKKSLRQ}(\mathrm{pS})$ FRRMRRSRV ${ }^{662}$ & $14.1 \pm 1.9$ \\
\hline $\operatorname{Lg} 12:$ & SRVKS LKKSLRQSFRRMRRSRV ${ }^{662}$ & $>100$ \\
\hline
\end{tabular}

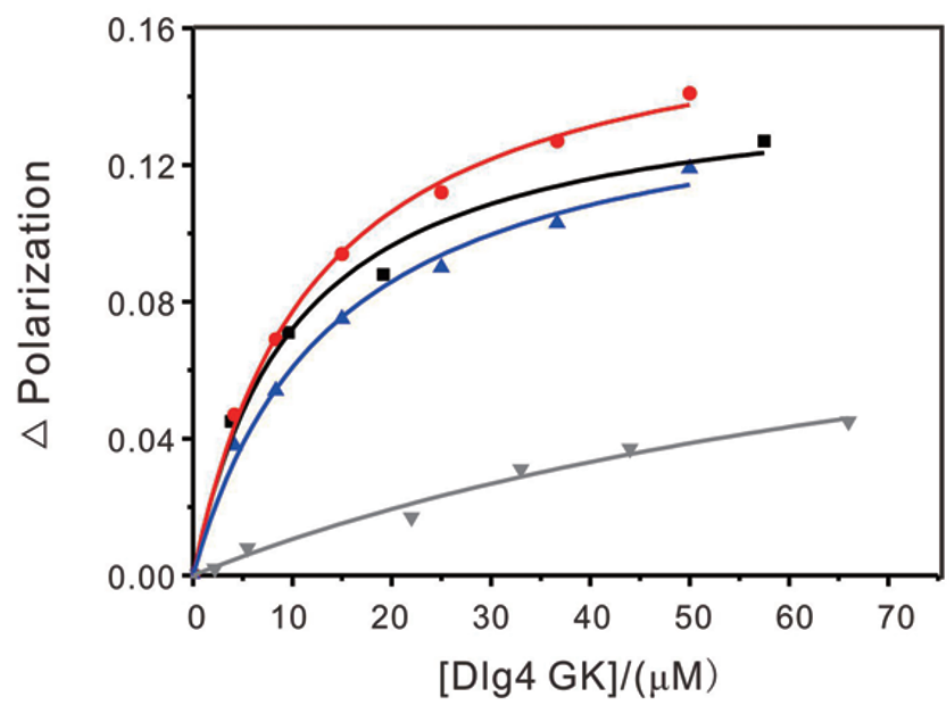

D

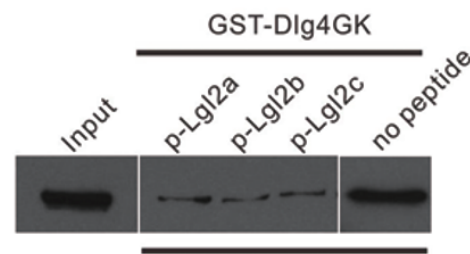

HA-Lgl2+aPKC

Figure 2 Phosphorylation of conserved Serine at the central linker region of Lgl2 is required for Dlg4/Lgl2 interaction. (A, B) GST pull-down-based assay of the binding of GST-tagged Dlg4 GK to various Lgl2 mutants. All Lgl2 mutants were coexpressed with Flag-tagged aPKC. (C) Fluorescence polarization-based measurement of the binding affinities of Dlg4 GK to the synthetic phosphor-Lgl2 peptides. The sequences of the peptides are shown at the top of the panel. (D) p-Lgl2 peptides block the binding of Lgl2 to Dlg4 GK. Equal amount of p-Lgl2 peptides (p-Lgl2a, p-Lgl2b and p-Lgl2c) were individually added into the mixture of HA-Lgl2 lysate and GST-DIg4 GK.

suggesting that phosphorylation of Lgl2 is essential for its interaction with Dlg.

To further consolidate the above conclusion, we used three synthetic phosphor-peptides with overlapping se- quences covering the aPKC phosphorylation region of Lg12 (p-Lgl2a, p-Lgl2b and p-Lgl2c; see Figures 1A and $2 \mathrm{C}$ for their detailed sequences) to measure their binding affinities to Dlg4 GK using fluorescence spectroscopy. 
Entirely consistent with the data obtained from the pulldown assay, each of the three phosphor-Lgl2 peptides binds to Dlg4 GK with comparable affinities (Kd: 10.2$14.1 \mu \mathrm{M}$; Figure 2C). As expected, unphosphorylated Lg12 peptide showed no detectable binding to Dlg4 GK (Figure 2C). We further performed the phosphor-peptide competition assay to compare the role of each phosphorylation site in GK binding. As shown in Figure 2D, the binding between HA-Lgl2 and Dlg4 GK dramatically decreased when each of $p$-Lgl2 peptides was added. $p$ Lg12a, p-Lg12b and p-Lg12c showed similar effect in competing with the full-length $\operatorname{Lg} 12$ to bind to Dlg4 GK, indicating that the three phosphorylation sites are likely to play equivalent roles in binding to Dlg4 GK. Collectively, the data shown in Figure 2 demonstrate that phosphorylation of any one of the three conserved Ser residues in the central linker region of $\mathrm{Lgl} 2$ by aPKC is sufficient for its binding to Dlg4.

\section{Overall structures of the Dlg4 GK/p-Lgl2 complexes}

Having established that phosphorylation of any one of the three Ser residues in Lgl2 results in its binding to Dlg4 GK, we next wanted to understand the molecular basis governing the phosphor-Lgl2/Dlg4 GK interaction. We tried to crystallize Dlg4 GK in complex with each of the three phosphor-Lgl2 peptides, and succeeded in solving the complex structures of Dlg4 GK/p-Lgl2a and Dlg4 $\mathrm{GK} / \mathrm{p}-\mathrm{Lgl} \mathrm{c}$ at the resolution of $2.0 \AA$ and $2.8 \AA$, respectively (Table 1).

In both of the complex structures, the Dlg4 GK domain adopts a conformation highly similar to that from the Dlg1/Dlg4 SH3-GK tandem structures solved earlier, comprising the GMP-binding subdomain, the LID subdomain and the Core subdomain [20, 23, 24] (Figure 3A, $3 \mathrm{~B}$ and $3 \mathrm{E}$ ). Both of the $\mathrm{p}-\mathrm{Lgl} 2 \mathrm{a}$ and $\mathrm{p}-\mathrm{Lgl2c}$ peptides adopt a short $\alpha$-helical conformation in the complex, occupying the concave groove clamped by the GMPbinding subdomain $(\beta 3, \beta 4, \beta 5$ and $\beta 6)$ and the Core subdomain ( $\alpha 1$ helix) with the same orientation. The p-Lgl2binding groove of GK has also been shown to bind to the phosphor-LGN peptide [20]. The bindings of $\mathrm{p}-\mathrm{Lgl} 2 \mathrm{a}$ and $\mathrm{p}-\mathrm{Lg} 12 \mathrm{c}$ to Dlg4 GK result in the burial of $\sim 542.3 \AA^{2}$ and $551.5 \AA^{2}$ of exposed surface area in the GK domain, respectively (Figure 3A-3D). Except for the N-terminal first residue and the $\mathrm{C}$-terminal last four residues of $\mathrm{p}$ Lgl2c, the electron densities of the rest of the phosphorLgl2 peptides are clearly defined in the complexes (Figure 3C and 3D). Notably, the phosphor-peptide binding only induces minor conformational changes of GK (pairwise RMSD values of $0.45 \AA$ and $0.57 \AA$, respectively, between apo-GK and GK in complex with p-Lgl2a and $\mathrm{p}-\mathrm{Lg} 12 \mathrm{c}$ ) (Figure 3E). Additionally, there are very little differences between the structures of Dlg4 GK in the two complex structures (RMSD value of $0.43 \AA$, Figure $3 E$ ).

\section{The interfaces of the Dlg4 GK/p-Lgl2 complexes}

The bindings of Dlg4 GK to p-Lgl2a and p-Lgl2c peptides share similar features, mainly via extensive polar (charge-charge and hydrogen bonding) and hydrophobic interactions. The phosphor-Ser (pSer0) from each of the two p-Lgl2 peptides forms a network of hydrogen bonds with Arg568, Arg571 from the $\beta 3 / \beta 4$-loop, Tyr580 in $\beta 3$ and Tyr609 in $\beta 6$ of the GK domain (Figure 4A and 4B). These interactions are further reinforced by a pair of intramolecular salt bridges formed between $\mathrm{R} 568_{\mathrm{GK}}$ and E574 $4_{\mathrm{GK}}$. Mutations of these pSer0-binding residues in GK totally disrupted the interaction between $\mathrm{p}-\mathrm{Lgl} 2$ and Dlg4 GK (Figure 4C). The hydrophobic residues at the +1 and +4 positions of the $\mathrm{p}$-Lgl2 peptides form extensive hydrophobic contacts with a conserved "hydrophobic helix binding groove" in GK formed by Pro564, Gly602, Tyr580, Tyr609 and Thr611 (Figure 4D). Substitution Gly602 of GK with Phe led to an $~ 4$-fold decrease of its binding to $\mathrm{p}$-Lgl2a, presumably due to the steric hindrance introduced by the bulky side chain of Phe from the mutant (Figure 4C and 4D). Besides these common binding features, there exist some unique interactions between GK and each of the two p-Lgl2 peptides. The side chain of Phe $654_{\mathrm{p}-\mathrm{Lg} 2 \mathrm{a}}$ in the +9 position inserts into a hydrophobic cradle [21] formed by the side chains of Ala601 and Gln603 from $\beta 5$, Leu608 from $\beta 6$ and Ile593 from $\alpha 2$ of GK (Figure 4A and 4D). Note that similar hydrophobic interactions also exist in the Dlg1 SH3-GK/pLGN complex (Figure 4D), and the hydrophobic cradle only exists in the Dlg subfamily of MAGUK proteins $[20,21]$. Substitution of Ala601 with Phe, which should decrease the hydrophobic pocket size, led to a 2-fold decease in GK's binding to p-Lgl2a (Figure 4C and 4D). The $\mathrm{p}-\mathrm{Lg} 12 \mathrm{c}$ peptide lacks the equivalent hydrophobic residue at the +9 position. Instead, it contains two positively charged residues (Arg at the -2 position and Lys at the -5 position, Figure 4D), which form a hydrogen bonding network with Arg578, Asp579 and Ser561 from GK (Figure 4B).

\section{Comparison of the GK/p-Lgl2 and GK/p-LGN complex structures}

We next compared the GK/p-Lgl2 complex structures with our recently reported GK/p-LGN structure [20], hoping to derive a general binding mechanism between Dlg GK and its phosphor-target peptides. The target recognition groove of the GK domain contains three distinct binding regions: a phosphor-Ser recognition site composed of Arg568, Arg571, Glu574, Tyr580 and Tyr609 in 
Table 1 Statistics of X-ray crystallographic data collection and model refinement

\begin{tabular}{|c|c|c|}
\hline Data set & Dlg4 GK/p-Lgl2a & Dlg4 GK/p-Lgl2c \\
\hline \multicolumn{3}{|l|}{ Data collection } \\
\hline Space group & $P 3,21$ & $P 6_{3} 22$ \\
\hline \multicolumn{3}{|l|}{ Cell dimensions } \\
\hline $\mathrm{a}, \mathrm{b}, \mathrm{c}(\AA)$ & $\mathrm{a}=84.082, \mathrm{~b}=84.082, \mathrm{c}=65.883$ & $\mathrm{a}=161.518, \mathrm{~b}=161.518, \mathrm{c}=45.032$ \\
\hline Resolution range $(\AA)$ & $50.00-2.04(2.08-2.04)$ & $50.00-2.80(2.85-2.80)$ \\
\hline No. of unique reflections & $17523(864)$ & $8915(424)$ \\
\hline Redundancy & $7.1(6.9)$ & $9.8(10.2)$ \\
\hline $\mathrm{I} / \sigma$ & $35.2(6.5)$ & $48.2(5.4)$ \\
\hline Completeness $(\%)$ & $99.9(99.4)$ & $99.8(100.0)$ \\
\hline $\mathrm{R}_{\text {merge }}(\%)$ & $6.6(34.1)$ & $7.6(58.4)$ \\
\hline B_Wilson & 36.5 & 68.5 \\
\hline \multicolumn{3}{|l|}{ Structure refinement } \\
\hline Resolution $(\AA)$ & $42.05-2.04(2.17-2.04)$ & $42.86-2.80(3.21-2.80)$ \\
\hline \multirow[t]{2}{*}{$\mathrm{R}_{\text {work }} / \mathrm{R}_{\text {free }}(\%)$} & $16.57 / 20.18$ & $19.92 / 26.84$ \\
\hline & $(19.92 / 25.35)$ & $(26.69 / 31.68)$ \\
\hline rmsd bonds $(\AA) /$ angles $\left({ }^{\circ}\right)$ & $0.011 / 1.210$ & $0.009 / 1.150$ \\
\hline \multicolumn{3}{|l|}{ No. of reflections } \\
\hline Working set & 16606 & 8483 \\
\hline Test set & 888 & 425 \\
\hline No. of Protein (Peptide) atoms & $1448(125)$ & $1433(108)$ \\
\hline No. of solvent atoms & 191 & 24 \\
\hline \multicolumn{3}{|l|}{ Average B factor } \\
\hline Protein (main chain) & $47.9(51.9)$ & $85.1(87.9)$ \\
\hline Peptide (main chain) & $41.9(42.5)$ & $71.5(73.4)$ \\
\hline Solvent & 54.4 & 92.4 \\
\hline \multicolumn{3}{|l|}{ Ramachandran plot } \\
\hline Favored regions $(\%)$ & 97.94 & 97.87 \\
\hline Allowed (\%) & 2.06 & 2.13 \\
\hline Outliers (\%) & 0 & 0 \\
\hline
\end{tabular}

Numbers in parentheses represent the value for the highest resolution shell. $R_{\text {merge }}=\sum\left|I_{i}-I_{m}\right| / \sum I_{i}$, where $I_{i}$ is the intensity of the measured reflection and $\mathrm{I}_{\mathrm{m}}$ is the mean intensity of all symmetry related reflections. $\mathrm{R}_{\text {work }}=\Sigma|| \mathrm{F}_{\mathrm{obs}}|-| \mathrm{F}_{\text {calc }}|||\Sigma| \mathrm{F}_{\mathrm{obs}} \mid$, where $\mathrm{F}_{\mathrm{obs}}$ and $\mathrm{F}_{\text {calc }}$ are observed and calculated structure factors. $\mathrm{R}_{\text {free }}=\Sigma_{\mathrm{T}}|| \mathrm{F}_{\text {obs }}|-| \mathrm{F}_{\text {calc }}|| \Sigma_{\mathrm{T}}\left|\mathrm{F}_{\text {obs }}\right|$, where $\mathrm{T}$ is a test data set of about $5 \%$ of the total reflections randomly chosen and set aside prior to refinement.

Dlg4 GK (referred to as "the phosphor-site") (Figure 4D and 4E; highlighted in yellow); a hydrophobic groove formed by Pro564, Gly602, Tyr604, Tyr609 and Thr611 that recognizes the two hydrophobic residues at the +1 and +4 positions from the target helix; and a hydrophobic cradle at the back side of $\beta 5$ formed by Ile593, Ala601, Leu608 and Gln603 in Dlg4 GK that interacts with a hydrophobic residue at the +9 position of the target peptide (Figure 4D and 4E). Compared to the p-LGN peptide, the $\mathrm{p}-\mathrm{Lg} 12$ peptides either lacks an Arg residue at the -2 position (e.g., the $\mathrm{p}$-Lgl2a peptide) or a hydrophobic residue at the +9 position (e.g., the p-Lgl2c peptide), explaining why $p$-Lgl2 peptides bind to Dlg4 GK less strongly than the p-LGN peptide [20] (Figures 2C, 4D and 4E).

Phosphorylation of Lgl2 plays a role in the polarization of epithelia

We reasoned that, if the phosphorylation-dependent interaction between $\mathrm{Lgl}$ and $\mathrm{Dlg}$ is functionally important, abolishment of Lg12 phosphorylation will result in polarization defects in MDCK cells. In a calcium switch assay, Lg12 knockdown caused defects of tight junction formation, indicating that $\mathrm{Lg} 12$ plays a role in the process of tight junction formation and cell polarity establishment in MDCK cells (Figure 5A and 5B). The wild-type 

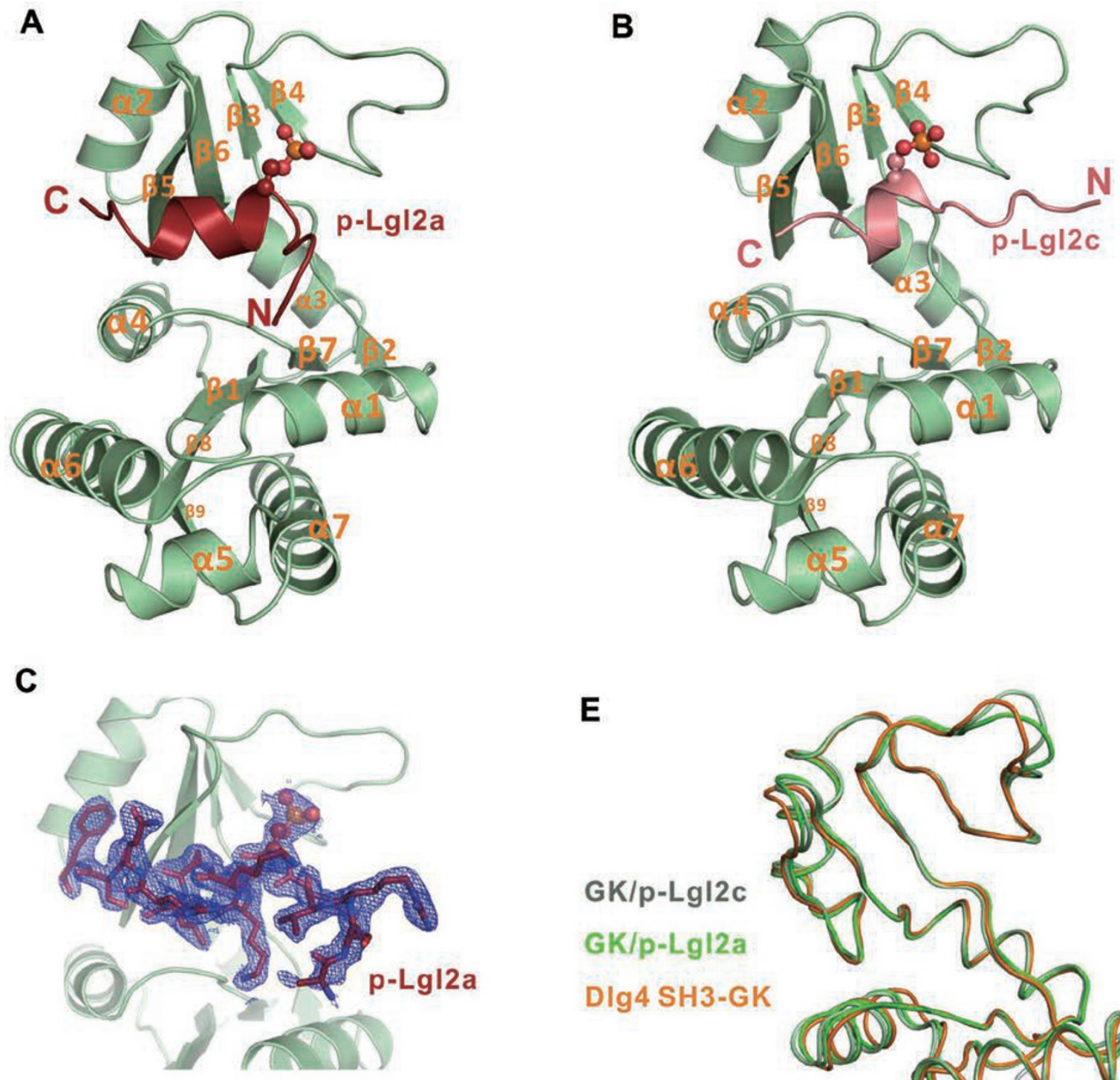

E
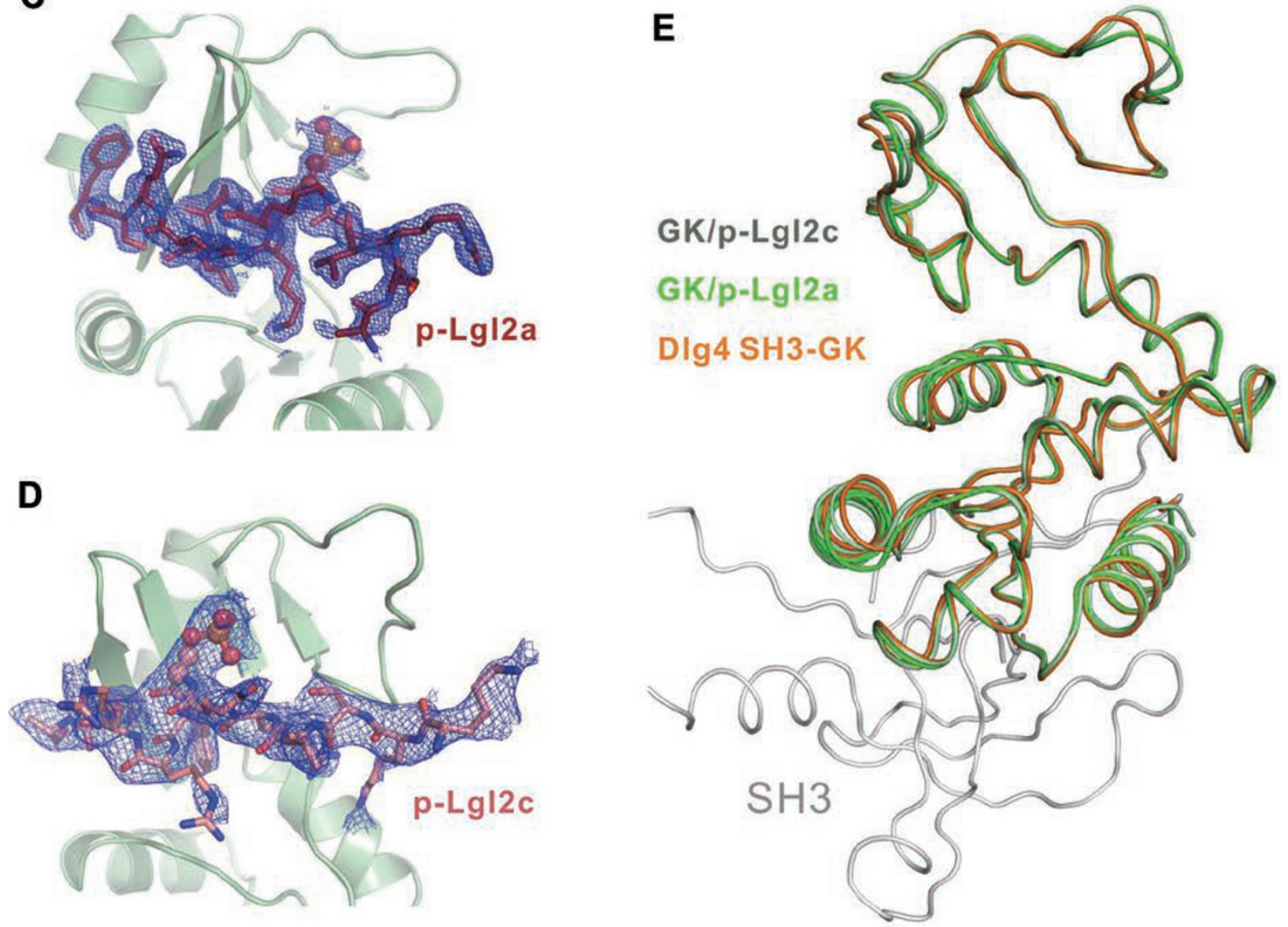

Figure 3 The overall structures of Dlg4 GK in complex with the p-Lgl2a and p-Lgl2c peptides. (A, B) Ribbon diagram representation of the Dlg4 GK/p-Lgl2a complex (A), and the Dlg4 GK/p-Lgl2c complex (B). Phosphate group of each peptide is presented in the stick and ball model. (C, D) 2Fc-Fo simulated omit map of the p-Lgl2a peptide (C) and the p-Lgl2c peptide (D) in the complexes countered at the level of 1.0 o. (E) Superposition of the structures of Dlg4 GK from GK/p-Lgl2a (deep green), GK/p-Lgl2c (light green) and the SH3-GK tandem (orange, PDB code: 1KJW). 
A

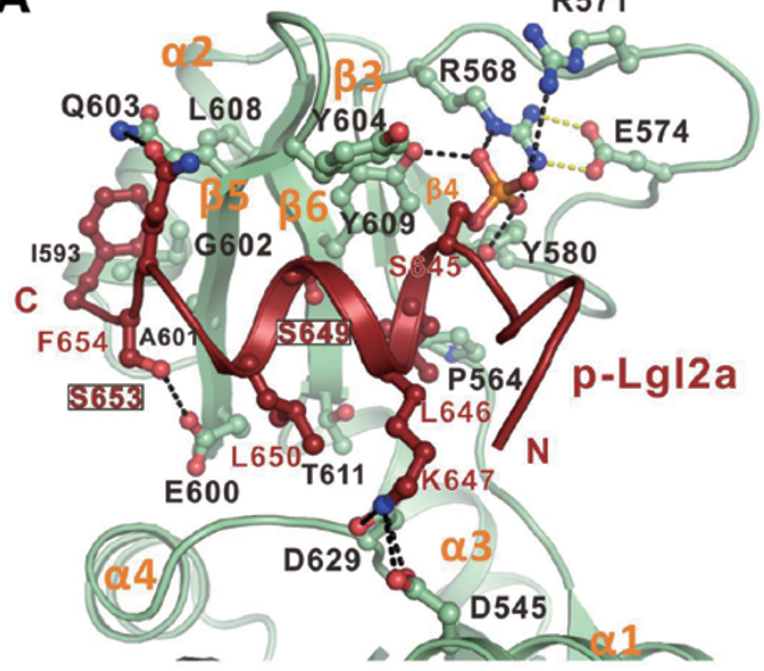

B

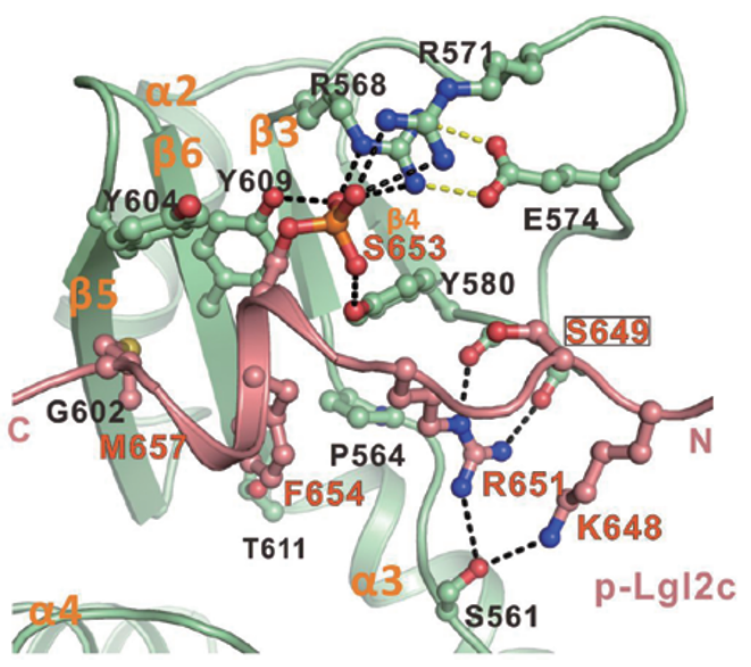

C

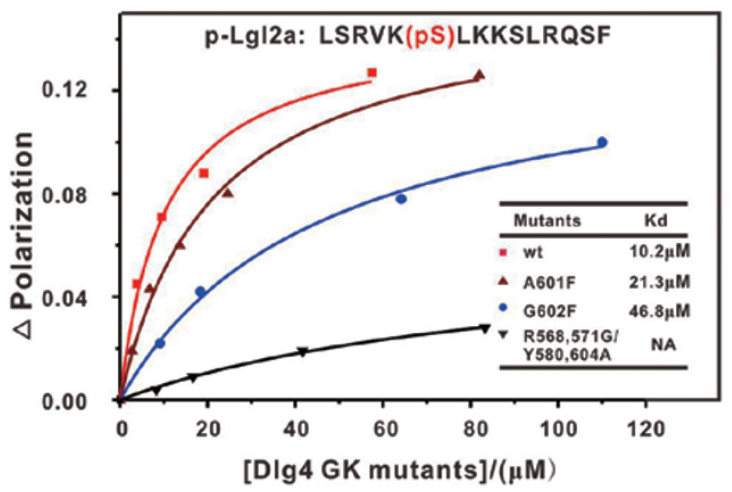

D

Dhosphor-site

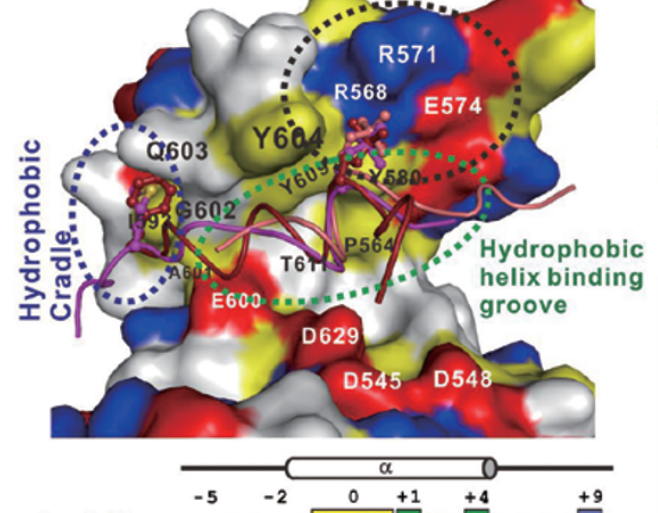

d1 Hydrophobic helix

p-Lgl2a: LSRVK (pS) LKKSLRQS

p-Lgl2c:KKSLRQ(pS) FRRMR

P-IGN : RRH (pS) MENL--EL KLTP



E

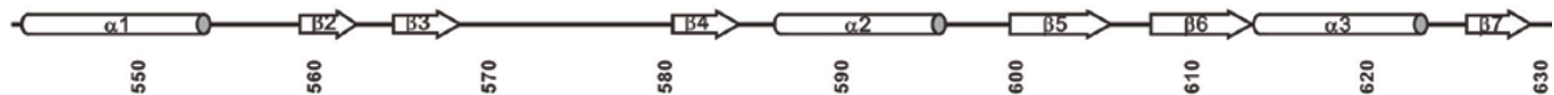

rDlg4 TKDRANDDLLSEFPDKFGSCVPHTTRPKREYEIDGRDYHFVSSREKMEKDIQAHKFIEAGQYNSHLYGTSVQSVREVAEQGKHCILDV

hDlg1 MKDRINDDIISEFPDKEGSCVPHTTRPKRDYEVDGRDYHFVTSREQMEKDIQEHKFIEAGQYNNHLYGTSVQSVREVAEKGKHCILDV

hD1g2 MKDRINDDLISEFPDKFGSCVPHTTRPKRDYEVDGRDYHFVISREQMEKDIQEHKFIEAGQYNDNLYGTSVQSVRFVAERGKHCILDV

hDlg3 MKDRVNDDLISEFPHKFGSCVPHTTRPRRDNEVDGQDYHFVVSREQMEKDIQDNKFIEAGQFNDNLYGTSIQSVRAVAERGKHCILDV

hDlg4 TKDRANDDLLSEFPDKFGSCVPHTTRPKREYEIDGRDYHFVSSREKMEKDIQAHKFIEAGQYNSHLYGTSVQSVREVAEQGKHCILDV

dDIg LKDRINDDLISEYPDKFGSCVPHTTRPKREYEVDGRDYHFVSSREQMERDIQNHLFIEAGQYNDNLYGTSVASVREVAEKGKHCILDV

CDIg LKDRINDELVNRDPSKFSSCVPHTSRP PREGEVNGRDYYFVN - KHNMEEDVKNNLFIEAGQFQNNLYGTSIQSVRDVANQGRHCILDV

p-Lgl2a :

p-Lgl2c :

p-LGN :

$\begin{array}{llllll} & \bullet & \bullet & & \bullet & \bullet \\ \star & \star & \star & \star & \star & \star \star \star \\ \mathbf{\Delta} & \boldsymbol{\Delta} & \boldsymbol{\Delta} & \boldsymbol{\Delta} & \boldsymbol{\Delta} & \boldsymbol{\Lambda} \mathbf{\Delta}\end{array}$

$\bullet \bullet \bullet \bullet \bullet$
$\star \star$
$\Delta \quad \Delta \Lambda \Lambda$

-๑

$\star \star$

$\Delta \mathbf{\Delta}$

Figure 4 Detailed interactions between Dlg4 and Lgl2. (A, B) The interaction interfaces between Dlg4 GK and p-Lgl2a (A), and p-Lgl2c (B). (C) Summary of the quantitative binding constants between various DIg4 GK mutants and the p-Lgl2a peptide. All quantitative binding data were derived from fluorescence-based titration assays. (D) Comparison of the binding modes of p-Lgl2a, p-Lgl2c and p-LGN to DIg GK. The structure-based sequence alignment of these three phosphor-peptides is shown below. (E) Structure-based sequence alignment of Dlg GK from different species (rDlg, rat Dlg; hDlg, human Dlg; dDIg, Drosophila Dlg; cDlg, C. elegans Dlg). In GK domains, the residues in the phosphor-site, the hydrophobic groove and the hydrophobic cradle are highlighted in yellow, green and blue, respectively. Residues involved in binding to p-LGN, p-Lgl2a and $\mathrm{p}-\mathrm{LgI} 2 \mathrm{c}$ are annotated below as triangles, dots and stars, respectively. 
A

MDCK II

KD Lgl2

$\left.\mathrm{KD} \mathrm{Lg}\right|_{2}+\mathrm{HA}-\left.\left.\mathrm{Lg}\right|_{2} \quad \mathrm{KD} \mathrm{Lg}\right|_{2}+\mathrm{HA}-\left.\mathrm{Lg}\right|_{2}-3 \mathrm{~A}$

ZO-1
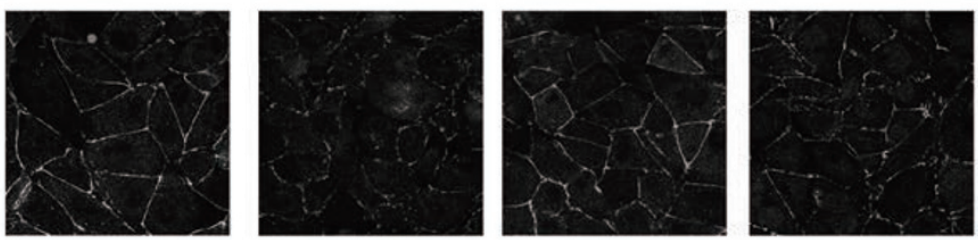

Merge
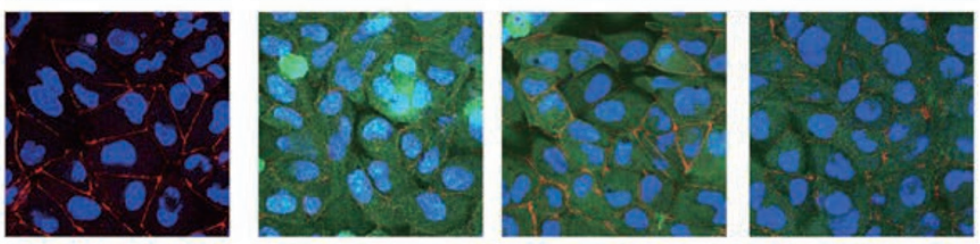

B

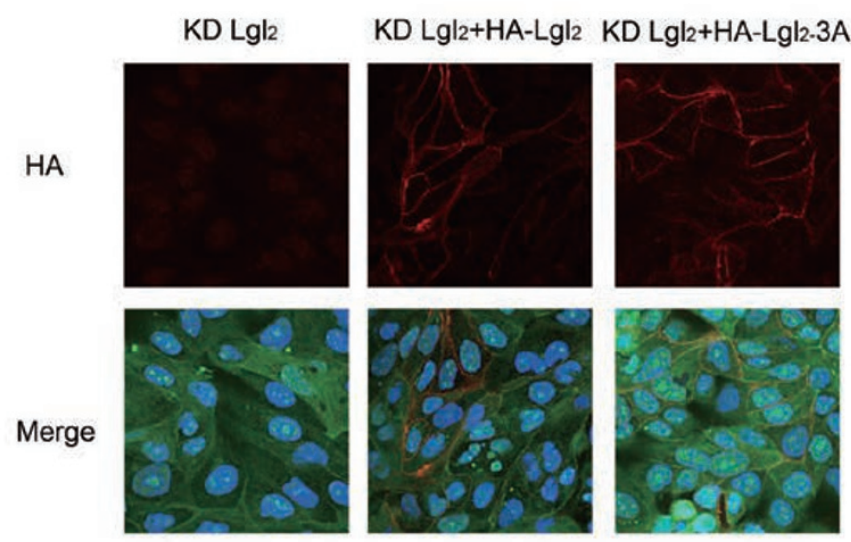

C

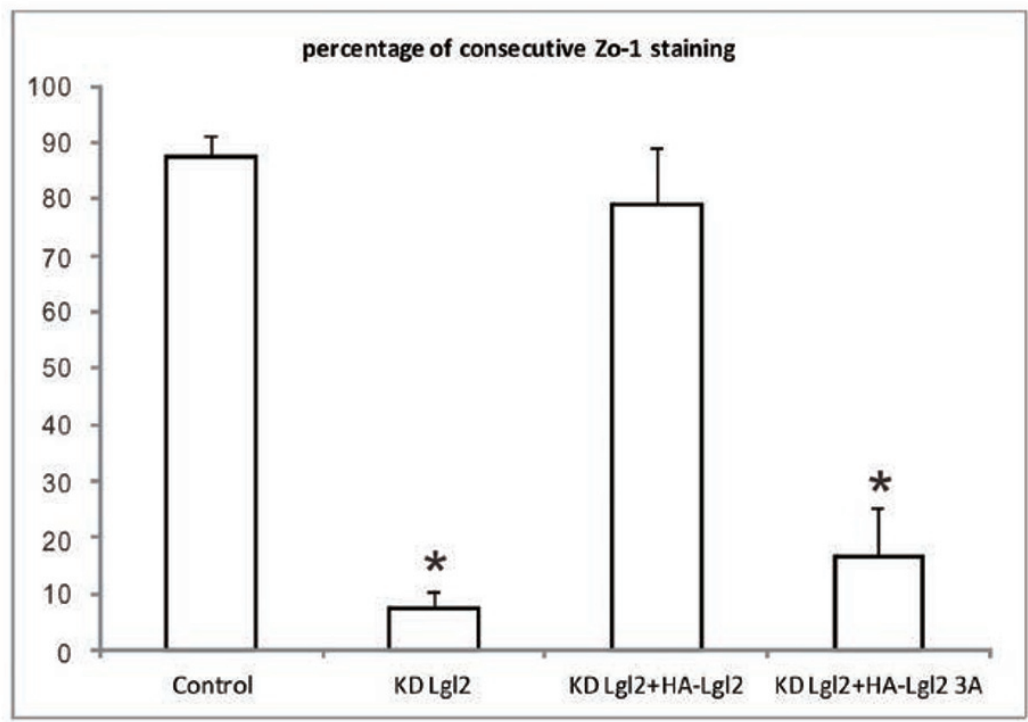

Figure $5 \mathrm{Lg}$ |2 3A mutant cannot rescue the tight junction formation defect caused by the loss of endogenous Lgl2 in MDCK cells. (A) Lgl2 3A mutant did not rescue the tight junction formation defects caused by loss of Lgl2 during a calcium switch. MDCK II cells with or without stable knockdown of Lgl2 were transfected with HA-tag only vector, HA-WT-Lgl2 or HA-Lgl23A. Cells were subjected to a calcium switch and stained for ZO-1 at $1 \mathrm{~h}$ after re-addition of calcium. (B) The expression of ectopic Lgl2 or Lgl2-3A in A are shown by immunofluorescence with the anit-HA antibody. (C) Quantification of percentage of consecutive ZO-1 staining in A. Error bars indicate SD values. ${ }^{*} P<0.001$ when compared with the control. 
HA-Lg12, but not the phosphorylation-defective mutant of Lg12 (HA-Lgl2 3A), can rescue the tight junction defects caused by loss of endogenous Lg12 in the calcium switch assay (Figure 5). These data suggest that phosphorylation of Lgl2 is critical for its function in the establishment of apical-basal polarity in MDCK cells.

\section{Discussion}

Proper apical-basal cell polarity, involved in diverse processes regulated by highly organized evolutionarily conserved polarity factors, is extremely important for normal epithelial tissue morphology. Several sets of evolutionarily conserved polarity complexes, including the Scribble, Par and Crumbs complexes, communicate with each other to define the different functional domains of epithelial cells. Unlike the Par and Crumbs complexes which have been extensively studied in the molecular details governing their complex formations, very little is known about how the Scribble complex is assembled physically. Our biochemical and structural studies presented in this work provides the first glimpse of the molecular mechanism underlying the interaction between Dlg and Lgl. We demonstrate that the interaction between Lgl and Dlg strictly depends on the phosphorylation of one or more of the three conserved Ser residues in the central linker region of Lgl. Amino-acid sequence alignment shows that the residues that are required for the interaction are completely conserved both in $\mathrm{Lgl}$ and in Dlg from worm to human (Figures $1 \mathrm{~A}$ and $4 \mathrm{E}$ ), indicating that this phosphorylation-dependent Lgl/Dlg interaction is a conserved property during the evolution.

Although our biochemical data demonstrated that each of the three phosphor-Lgl peptides can bind to one equivalent Dlg GK with comparable affinity (Figure 2C and 2D), it is unclear how the binding stoichiometry would be when multiple Ser residues in Lgl are phosphorylated. A careful analysis of the structures of the $\mathrm{Dlg} \mathrm{GK} / \mathrm{p}-\mathrm{Lgl}$ complexes presented in this work and the Dlg SH3-GK/p-LGN complex structure solved earlier [20] allows us to deduce that only a 1:1 Dlg/Lgl complex can be formed even when 2 or 3 Ser residues in Lgl are phosphorylated. It is noted that the three Ser residues in the Lgl central linker are evenly spaced by a threeresidue gap (Figure 1A). The structures of the Dlg GK/ p-Lgl and the Dlg SH3-GK/p-LGN complexes, together with a detailed biochemical analysis, showed that the residues at the +4 and +9 positions (i.e., 4 and 9 residues C-terminal to the pSer0; Figure 4D) of the target peptides are critically important for the hydrophobic packing, and the residues in these two positions cannot be charged. On the other hand, the residue at the -4 or -9 positions of the target peptides do not play important roles in binding to Dlg GK (Figure 4). Therefore, when any of the two Ser residues in Lgl are phosphorylated, Dlg GK can only bind to the most C-terminal p-Ser residue and form a 1:1 stoichiometric complex (Figure 6A, a2-a4). By the same logic, when all three Ser residues are phosphorylated, Dlg GK can only engage the last p-Ser (Figure 6A, a5).

The localization of $\mathrm{Lgl}$ in epithelia is tightly regulated by its phosphorylation status, whereas Dlg and Scribble are constitutively localized to the lateral membranes [9, 25]. When unphosphorylated, apical cell cortex-localized Lgl can interact with and inhibit the enzyme activity of aPKC $[15,26]$. Upon phosphorylation, Lgl undergoes conformational changes causing its dissociation from the apical cortex and concomitant release of its inhibition on aPKC, and subsequent accumulation at the basal-lateral membranes [26, 27]. Notably, expression of an unphosphorylatable $\mathrm{Lgl}(\mathrm{Lgl} 23 \mathrm{~A})$ led to severe defects in the segregation of cell fate determinants in the asymmetric cell division of Drosophila neuroblasts [28-30], probably due to constitutive inhibition of aPKC at the apical cortex. In MDCK cells, the Lg12 3A mutant was shown to localize at the apical membrane and result in the severe cell polarity defects, whereas the wild-type Lgl mainly localizes at the basal-lateral region [31]. In a calcium switch assay, defects of tight junction formation caused by Lg12 knockdown could be rescued by wild-type HALg12 but not the HA-Lgl2 3A mutant, further indicating that phosphorylation of $\mathrm{Lgl} 2$ is critical for its function in the establishment of cell polarity (Figure 5). Our biochemical and structural work presented here provides a mechanistic explanation for these observations (Figure $6 \mathrm{~B})$. aPKC-mediated phosphorylation is likely to play an instructive role by switching on the direct interaction between Dlg and Lgl. Since Dlg is constitutively localized at the basal-lateral membranes in polarized epithelia, the phosphorylated Lgl is then also sequestered to the basallateral membranes to play a role in the polarization of epithelia (Figure 6B). At this point, it is still not clear how Scribble is physically assembled with the $\mathrm{Dlg} / \mathrm{p}-\mathrm{Lgl}$ complex.

In summary, the data presented in the current study have demonstrated that the two tumor suppressors, Dlg and $\mathrm{Lgl}$, directly interact with each other in a phosphorylation-dependent manner. In addition to answering the long-standing question regarding the Scribble complex assembly (although partially), our study also provides valuable insights into the design of possible small molecules targeting the phosphorylation-dependent $\mathrm{Dlg} / \mathrm{Lgl}$ interaction for future functional studies of the $\mathrm{Dlg} / \mathrm{Lgl}$ complex in various biological processes including tissue development and cancer formation. 
A

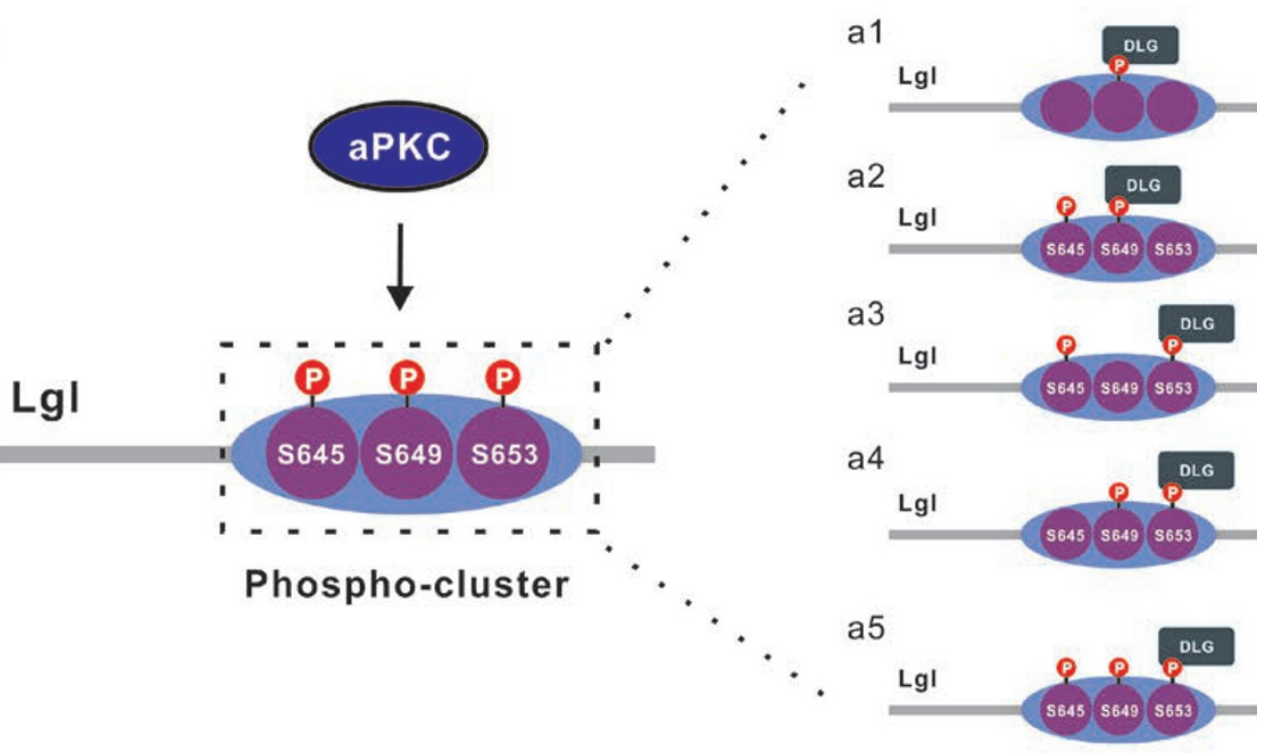

B



Figure 6 A schematic model showing the phosphorylation-dependent recruitment of Lgl by basal-lateral localized Dlg. (A) Lgl contains three possible aPKC phosphorylation sites (S645, S649 and S653). Among the five possible phosphorylation patterns, Dlg GK can only bind to one phosphor-Ser, forming a 1:1 Dlg/Lgl complex. (B) In the unphosphorylated state, Lgl can form a complex with Par-6/aPKC at the apical cortex and inhibits the activity of aPKC. Once phosphorylated by aPKC, $\mathrm{Lgl}$ is released from the apical cell cortex. Phosphorylated $\mathrm{Lgl}$ is then recruited to the basal-lateral membranes via direct binding to Dlg, forming the basal-lateral DIg/Lgl complex. 


\section{Materials and Methods}

\section{Protein preparation}

The rat Dlg4 GK domain (residues aa 531-713) and various mutants (Figure 4C) were individually cloned into a modified version of pET15b vector. All the mutations were created using the standard PCR-based method and confirmed by DNA sequencing. Recombinant proteins were expressed in Escherichia coli BL21 (DE3) host cells at $16{ }^{\circ} \mathrm{C}$ and were purified by $\mathrm{Ni}^{2+}-\mathrm{NTA}$ agarose affinity chromatography followed by size-exclusion chromatography. For GST pull-down experiments, Dlg4 GK was fused to the C-terminus of GST using the pGEX-4T-1 vector and purified by GSH-Sepharose affinity chromatography.

\section{GST pull-down assay}

For GST pull-down assay, GST-tagged Dlg4 GK was first loaded to $30 \mu \mathrm{l} \mathrm{GSH}$-sepharose 4B slurry beads in an assay buffer (50 $\mathrm{mM}$ Tris, $\mathrm{pH}$ 8.0, $100 \mathrm{mM} \mathrm{NaCl}, 1 \mathrm{mM}$ DTT and $1 \mathrm{mM}$ EDTA). The GST fusion protein loaded beads were then incubated for $2 \mathrm{~h}$ at $4{ }^{\circ} \mathrm{C}$ with lysates of HEK293T cells expressing various forms of HA-tagged full-length human $\mathrm{Lgl} 2$ with or without co-expression of Flag-tagged aPKC. After three times washing, the proteins captured were eluted by boiling, resolved by $10 \%$ SDS-PAGE, and detected by western blot with specific antibodies (anti-pLgl, 1:500; Thermo Scientific).

\section{Fluorescence polarization assay}

Fluorescence assay was performed on a PerkinElmer LS-55 fluorimeter equipped with an automated polarizer at $25{ }^{\circ} \mathrm{C}$. Commercially synthesized phosphor-Lgl2 peptides (Figure $2 \mathrm{C}$ ) were labeled with fluorescein-5-isothiocyanate (Invitrogen, Molecular Probe) at their N-termini. In a typical assay, the FITC-labeled peptide $(\sim 1 \mu \mathrm{M})$ was titrated with binding partners in a $50 \mathrm{mM}$ Tris (pH 8.0) buffer containing $100 \mathrm{mM} \mathrm{NaCl}, 1 \mathrm{mM}$ DTT and $1 \mathrm{mM}$ EDTA. The Kd values were obtained by fitting the titration curves with the classical one-site binding model.

\section{Crystallography}

Crystals of the Dlg4 GK/p-Lgl2a and Dlg4 GK/p-Lgl2c complexes were obtained by the hanging drop vapor diffusion method at $18{ }^{\circ} \mathrm{C}$. Freshly purified Dlg4 GK was concentrated to $0.5 \mathrm{mM}$ before a saturating amount of $\mathrm{p}-\mathrm{Lgl} 2 \mathrm{a}$ or $\mathrm{p}$-Lgl2c peptide was added. The GK/p-Lgl2a complex crystals were grown in $0.2 \mathrm{M}$ lithium chloride, 20\% PEG3350, and the GK/p-Lgl2c crystals were from $0.3 \mathrm{M}$ ammonium sulfate, $0.1 \mathrm{M}$ Bis-Tris, pH 6.0, 16\% PEG3350. Crystals were soaked in crystallization solution containing $30 \%$ glycerol for cryo-protection. Molecular replacement was used to solve the structures of the two complexes using the GK domain from the Dlg4 SH3-GK tandem structure (PDB code: $1 \mathrm{KJW}$ ) as the search model using the software suits of Phaser [32] in CCP4 [33]. Refinements were carried out using refmac [33] followed by phenix.refinement [34]. The p-Lgl2 peptides were manually built. Coot [35] was used for model buildings and modifications. The structural figures were prepared by PyMOL (www.pymol.org).

\section{Calcium switch assay, immunofluorescence and quantifica-} tion

Calcium switch experiments were performed as described [36]. After electroporation, MDCKII cells were plated in 24-well plate with DMEM supplemented with $10 \%$ FBS, $100 \mathrm{U} / \mathrm{ml}$ penicillin and streptomycin (Hyclone). After $24 \mathrm{~h}$, the cells formed a confluent monolayer. Cells were washed four times with PBS and incubated in DMEM without calcium (2.5 mM EDTA) for $2 \mathrm{~h}$. After all the cells became round-up, the cultures were switched to a normal medium for $1 \mathrm{~h}$. Cells were washed and fixed in 4\% PFA with $0.2 \%$ TX-100. After blocking with 5\% BSA in PBS for $1 \mathrm{~h}$, cells were incubated with primary and secondary antibodies. Primary antibodies were monoclonal anti-HA 12CA5 (1:1 000), monoclonal anti-ZO-1 (Zymed; Invitrogen, Carlsbad, CA, USA) (1:1 000). The secondary antibody used was Alexa Fluor 594 (Invitrogen) conjugated goat anti-mouse antibodies. Hoechst 33342 (Invitrogen) was used for DNA staining. pLV-mU6-EF1-GFP vector (Biosettia, San Diego, CA, USA) was used for lentivirus-mediated stable knockdown of Lgl2 in MDCK cells, as previously described [37].

\section{Accession codes}

The atomic coordinates of Dlg4/p-Lgl2a and Dlg4/p-Lgl2c complexes have been deposited to the Protein Data Bank under the accession codes $3 \mathrm{WP} 0$ and $3 \mathrm{WP} 1$, respectively.

\section{Acknowledgments}

We thank Shanghai Synchrotron Radiation Facility (SSRF) BL17U for X-ray beam time. This work was supported by grants from RGC of Hong Kong (663610, 663811, 663812, HKUST6/ CRF/10, SEG HKUST06, AoE/M09/12 and T13-607/12R) to MZ and National Institute of Health (GM079506) to QD. MZ is a Kerry Holdings Professor in Science and a Senior Fellow of IAS at HKUST.

\section{References}

1 Knoblich JA. Asymmetric cell division: recent developments and their implications for tumour biology. Nat Rev Mol Cell Biol 2010; 11:849-860.

2 St Johnston D, Ahringer J. Cell polarity in eggs and epithelia: parallels and diversity. Cell 2010; 141:757-774.

3 Bilder D. Epithelial polarity and proliferation control: links from the Drosophila neoplastic tumor suppressors. Genes Dev 2004; 18:1909-1925.

4 Humbert PO, Grzeschik NA, Brumby AM, Galea R, Elsum I, Richardson HE. Control of tumourigenesis by the Scribble/ Dlg/Lgl polarity module. Oncogene 2008; 27:6888-6907.

5 Humbert PO, Dow LE, Russell SM. The Scribble and Par complexes in polarity and migration: friends or foes? Trends Cell Biol 2006; 16:622-630.

6 Bilder D, Li M, Perrimon N. Cooperative regulation of cell polarity and growth by Drosophila tumor suppressors. Science 2000; 289:113-116.

7 Assemat E, Bazellieres E, Pallesi-Pocachard E, Le Bivic A, Massey-Harroche D. Polarity complex proteins. Biochim Biophys Acta 2008; 1778:614-630.

8 Gateff E, Schneiderman HA. Neoplasms in mutant and cultured wild-type tissues of Drosophila. Natl Cancer Inst Monogr 1969; 31:365-397.

9 Bilder D, Perrimon N. Localization of apical epithelial determinants by the basolateral PDZ protein Scribble. Nature 2000; 403:676-680. 
10 Stewart M, Murphy C, Fristrom JW. The recovery and preliminary characterization of $\mathrm{X}$ chromosome mutants affecting imaginal discs of Drosophila melanogaster. Dev Biol 1972; 27:71-83.

11 Mathew D, Gramates LS, Pachard M, et al. Recruitment of scribble to the synaptic scaffolding complex requires GUKholder, a novel DLG binding protein. Curr Biol 2002; 12:531539.

12 Brooks SP, Coccia M, Tang HR, et al. The Nance-Horan syndrome protein encodes a functional WAVE homology domain (WHD) and is important for co-ordinating actin remodelling and maintaining cell morphology. Hum Mol Genet 2010; 19:2421-2432.

13 Walsh GS, Grant PK, Morgan JA, Moens CB. Planar polarity pathway and Nance-Horan syndrome-like $1 \mathrm{~b}$ have essential cell-autonomous functions in neuronal migration. Development 2011; 138:3033-3042.

14 Kallay LM, McNickle A, Brennwald PJ, Hubbard AL, Braiterman LT. Scribble associates with two polarity proteins, Lg12 and Vang12, via distinct molecular domains. J Cell Biochem 2006; 99:647-664.

15 Hutterer A, Betschinger J, Petronczki M, Knoblich JA. Sequential roles of Cdc42, Par-6, aPKC, and Lgl in the establishment of epithelial polarity during Drosophila embryogenesis. Dev Cell 2004; 6:845-854.

16 Hoege C, Constantinescu AT, Schwager A, Goehring NW, Kumar P, Hyman AA. LGL can partition the cortex of onecell Caenorhabditis elegans embryos into two domains. Curr Biol 2010; 20:1296-1303.

17 Betschinger J, Mechler BM, Knoblich JA. The Par complex directs asymmetric cell division by phosphorylating the cytoskeletal protein Lgl. Nature 2003; 422:326-330.

18 Plant PJ, Fawcett JP, Lin DC, et al. A polarity complex of mPar-6 and atypical PKC binds, phosphorylates and regulates mammalian Lgl. Nat Cell Biol 2003; 5:301-308.

19 Yamanaka T, Horikoshi Y, Sugiyama Y, et al. Mammalian $\mathrm{Lgl}$ forms a protein complex with PAR-6 and aPKC independently of PAR-3 to regulate epithelial cell polarity. Curr Biol 2003; 13:734-743.

20 Zhu J, Shang Y, Xia C, Wang W, Wen W, Zhang M. Guanylate kinase domains of the MAGUK family scaffold proteins as specific phospho-protein-binding modules. EMBO J 2011; 30:4986-4997.

21 Zhu J, Shang Y, Chen J, Zhang M. Structure and function of the guanylate kinase-like domain of the MAGUK family scaffold proteins. Front Biol 2012; 7:379-396.

22 Johnston CA, Doe CQ, Prehoda KE. Structure of an enzymederived phosphoprotein recognition domain. PLoS One 2012; 7:e36014.

23 McGee AW, Dakoji SR, Olsen O, Bredt DS, Lim WA, Pre- hoda KE. Structure of the SH3-guanylate kinase module from PSD-95 suggests a mechanism for regulated assembly of MAGUK scaffolding proteins. Mol Cell 2001; 8:1291-1301.

24 Tavares GA, Panepucci EH, Brunger AT. Structural characterization of the intramolecular interaction between the $\mathrm{SH} 3$ and guanylate kinase domains of PSD-95. Mol Cell 2001; 8:13131325.

25 Dow LE, Brumby AM, Muratore R, et al. hScrib is a functional homologue of the Drosophila tumour suppressor Scribble. Oncogene 2003; 22:9225-9230.

26 Yamanaka T, Horikoshi Y, Izumi N, Suzuki A, Mizuno K, Ohno S. Lgl mediates apical domain disassembly by suppressing the PAR-3-aPKC-PAR-6 complex to orient apical membrane polarity. J Cell Sci 2006; 119:2107-2118.

27 Betschinger J, Eisenhaber F, Knoblich JA. Phosphorylationinduced autoinhibition regulates the cytoskeletal protein Lethal (2) giant larvae. Curr Biol 2005; 15:276-282.

28 Knoblich JA. Mechanisms of asymmetric stem cell division. Cell 2008; 132:583-597.

29 Prehoda KE. Polarization of Drosophila neuroblasts during asymmetric division. Cold Spring Harb Perspect Biol 2009; 1:a001388.

30 Atwood SX, Prehoda KE. aPKC phosphorylates Miranda to polarize fate determinants during neuroblast asymmetric cell division. Curr Biol 2009; 19:723-729.

31 Musch A, Cohen D, Yeaman C, Nelson WJ, RodriguezBoulan E, Brennwald PJ. Mammalian homolog of Drosophila tumor suppressor lethal (2) giant larvae interacts with basolateral exocytic machinery in Madin-Darby canine kidney cells. Mol Biol Cell 2002; 13:158-168.

32 McCoy AJ, Grosse-Kunstleve RW, Adams PD, Winn MD, Storoni LC, Read RJ. Phaser crystallographic software. J Appl Crystallogr 2007; 40:658-674.

33 Murshudov GN, Skubak P, Lebedev AA, et al. REFMAC5 for the refinement of macromolecular crystal structures. Acta Crystallogr D Biol Crystallogr 2011; 67:355-367.

34 Adams PD, Afonine PV, Bunkoczi G, et al. PHENIX: a comprehensive Python-based system for macromolecular structure solution. Acta Crystallogr D Biol Crystallogr 2010; 66:213221.

35 Emsley P, Lohkamp B, Scott WG, Cowtan K. Features and development of Coot. Acta Crystallogr D Biol Crystallogr 2010; 66:486-501.

36 Chen X, Macara IG. Par-3 controls tight junction assembly through the Rac exchange factor Tiam1. Nat Cell Biol 2005; 7:262-269.

37 Wan Q, Liu J, Zheng Z, et al. Regulation of myosin activation during cell-cell contact formation by Par3-Lgl antagonism: entosis without matrix detachment. Mol Biol Cell 2012; 23:2076-2091.

(Supplementary information is linked to the online version of the paper on the Cell Research website.) 